\title{
Reconstruction tridimensionnelle surfacique et implantologie extra-orale : à propos d'un cas clinique
}

\author{
Bodard $A G^{1,2}$, Salino $S^{2}$, Fortin $T^{1}$, Coudert $\mathrm{JL}^{1}$ \\ ${ }^{1}$ Service de Consultations et Traitements dentaires, Lyon, France \\ ${ }^{2}$ Centre régional de Lutte contre le Cancer Léon Bérard, Lyon, France \\ bodard@lyon.fnclcc.fr
}

L'utilisation d'implants extra-oraux pour la stabilisation des épithèses a contribué à améliorer la qualité de vie des patients. Le choix de l'émergence implantaire est parfois difficile, compte-tenu des impératifs esthétiques de la future épithèse. L'utilisation de logiciels de reconstruction tridimensionnelle, couplée à un guide chirurgical, permet une planification du geste et un choix plus facile du diamètre, de la longueur et de l'émergence des implants.

Un patient de 35 ans, traité à l'âge de 3 ans pour une tumeur de l'orbite droit par exentération est adressé pour une réhabilitation par épithèse implanto-portée. Une tentative de mise en place d'implants extra-oraux avait échoué. Trois implants avaient été mis en place : un implant ne s'est pas ostéointégré, un second a entraîné une sinusite frontale majeure ; seul le troisième a pu être laissé en place. Le patient a été adressé pour la prise en charge de sa sinusite, en collaboration avec les chirurgiens oto-rhino-laryngologistes.

Après traitement de la sinusite, la réhabilitation a pu reprendre. Un système de repositionnement non invasif avec un guide radiochirurgical, inspiré du système d'Alfano et al. (2005), associé à une reconstruction tridimensionnelle surfacique par le logiciel $\mathrm{MVS}^{\circledR}$ ont été mis en œuvre. Le système de repositionnement était composé d'un guide radiochirurgical, avec des marqueurs fiduciaires (gutta percha), d'un bras de liaison rigide et d'une gouttière rigide thermoformée maxillaire. La reconstruction a permis de mettre en évidence les zones d'implantation possibles, compte tenu des échecs antérieurs. Le calcul de l'émergence implantaire a pu être effectué et reporté sur le guide opératoire.

La reconstruction tridimensionnelle facilite le choix de l'émergence et du site implantaires. D'autres auteurs ont utilisé des guides stéréolithographiques (Nerdonck et al. 2003, Samartino et al. 2004), mais ceux-ci sont relativement lourds à mettre en œuvre et coûteux.

La plupart des systèmes de guides conventionnels utilisés jusqu'alors ont un inconvénient majeur, l'impossibilité d'intégrer les variables opératoires, et une limite le passage des informations du virtuel au réel.

La reconstruction tridimensionnelle surfacique est intéressante pour la mise en évidence de très petits éléments anatomiques, comme dans le cas clinique présenté, même si la reconstruction volumique a fait d'importantes avancées dans ce domaine. 